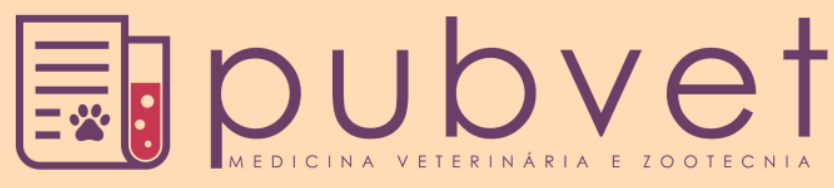

HTTP://DX.DOI.ORG/10.22256/PUBVET.V11N11.1085-1097

\title{
Expressão facial, vocalização e posturas anômalas em bovinos submetidos à eletroejaculação convencional
}

\author{
Jackson Barros do Amaral ${ }^{1 *}$, Rita Maria Ladeira Pires' ${ }^{1}$, Luís Alberto Ambrósio ${ }^{1}$, Flávia \\ Augusta de Oliveira ${ }^{2}$, Guilherme Trevisan ${ }^{3}$
}

${ }^{I}$ Centro de Análise e Pesquisa Tecnológica dos Agronegócios de Bovinos de Leite, Instituto de Zootecnia, Agência Paulista de Tecnologia dos Agronegócios, Secretaria de Agricultura e Abastecimento do Estado de São Paulo, Caixa Postal 60, CEP 13460-000, Nova Odessa, SP, e-mail jackson@iz.sp.gov.br

${ }^{2}$ Universidade Federal de Goiás - UFG, Jataí - Goiás, Brasil.

${ }^{3}$ Médico Veterinário, Mestrando da Faculdade de Medicina Veterinária e Zootecnia da Universidade Estadual Paulista "Júlio de Mesquita Filho”- Distrito de Rubião Jr., s/n - Botucatu/SP CEP 18618-970, e-mail guilherme_med.vet@live.com

*Autor para correspondência.

RESUMO. A eletroejaculação é utilizada rotineiramente para coletas de sêmen dos bovinos. Os estudos das alterações comportamentais em bovinos submetidos à eletroejaculação convencional são raros, não sendo elucidada a expressão facial neste procedimento. Este trabalho teve o objetivo de avaliar a expressão facial, vocalização e posturas anômalas em 62 touros submetidos à eletroejaculação, em exames andrológicos. Utilizou-se eletroejaculador convencional aplicando-se, sucessivamente, estímulos elétricos com intensidades crescentes de corrente contínua, até cinco estímulos para obtenção da ejaculação. As coletas foram conduzidas durante a eletroejaculação pelas filmagens dos animais contidos, focando a cabeça e o pescoço onde se avaliou a expressão facial, vocalização, posturas da cabeça, atitude de deitar, além da salivação e ejaculação. A expressão facial foi avaliada pelas alterações comportamentais da boca e dos olhos. Nos resultados das análises do método de regressão logística houve efeito significativo a 5\% de probabilidade quando se considerou a intensidade dos estímulos elétricos no procedimento da eletroejaculação nos registros das posturas da cabeça, dos olhos, na vocalização, na atitude de deitar e na ejaculação. Entretanto, não houve efeito significativo quando se considerou a intensidade dos estímulos elétricos no procedimento da eletroejaculação nos registros das alterações comportamentais da boca e na ocorrência de sialorreia. As posturas da cabeça foram $71,0 \%$ no primeiro estímulo elétrico; $82,3 \%$ no segundo estímulo; $85,48 \%$ no terceiro; $89,7 \%$ no quarto e $82,6 \%$ no quinto estímulo elétrico. As alterações comportamentais dos olhos apresentaram $74,2 \%$ no primeiro estímulo elétrico; $87,10 \%$ no segundo e terceiro estímulo; $98,3 \%$ no quarto e $97,8 \%$ no quinto estímulo elétrico. Nenhum animal deitou durante o primeiro estímulo elétrico. A atitude de deitar no segundo, terceiro, quarto e quinto estímulos elétricos foram $6,5 \% ; 4,8 \% ; 5,2 \%$ e $13,0 \%$, respectivamente. As vocalizações foram mais elevadas no quarto estímulo elétrico, com $12,1 \%$ e no quinto, com 10,9\%. Conclui-se que a eletroejaculação induziu alterações da expressão facial, vocalização e atitude de deitar durante o exame. Estes resultados sugerem a necessidade de refinamento da eletroejaculação, aprimoramento do protocolo de coleta de sêmen, dos equipamentos utilizados, das instalações e do manejo dos animais antes, durante e após este procedimento para melhoria do bem-estar animal.

Palavras chave: Bem-estar, coleta de sêmen, comportamento, exame andrológico, touro. 


\title{
Facial expression, vocalization and abnormal postures of cattle submitted to conventional eletroejaculation
}

\begin{abstract}
Electroejaculation is routinely used for bovine semen collections. Studies of behavioral changes in cattle submitted to conventional electroejaculation are rare, and facial expression in this procedure is not elucidated. The objective of this study was to evaluate the facial expression, vocalization and anomalous postures in 62 bulls submitted to electro - ejaculation, in andrological examinations. A conventional electro-jogger was used by successively applying electrical stimuli with increasing currents of direct current up to five stimuli to obtain ejaculation. The collections were conducted during the electroejaculation by the filming of the animals contained, focusing the head and neck where the facial expression, vocalization, head postures, lying attitude, salivation and ejaculation were evaluated. Facial expression was assessed by behavioral changes in the mouth and eyes. In the results of the analysis of the logistic regression method, there was a significant $5 \%$ probability effect when considering the intensity of the electrical stimuli in the electroejaculation procedure in the registers of head postures, eyes, vocalization, lying down and ejaculation. However, there was no significant effect when considering the intensity of electrical stimuli in the electroejaculation procedure in the records of behavioral changes of the mouth and in the occurrence of sialorrhea. The head postures were $71.0 \%$ in the first electrical stimulus; $82.26 \%$ in the second stimulus; $85.5 \%$ in the third; $89.66 \%$ in the fourth and $82.6 \%$ in the fifth electric stimulus. The behavioral alterations of the eyes presented $74.2 \%$ in the first electric stimulus; $87.1 \%$ in the second and third stimuli; $98.3 \%$ in the fourth and $97.8 \%$ in the fifth electric stimulus. No animal lay down during the first electric stimulus. The attitude of lying in the second, third, fourth and fifth electric stimuli were $6.5 \% ; 4.8 \% ; 5.2 \%$ and $13.0 \%$, respectively. Vocalizations were higher in the fourth electrical stimulus, with $12.1 \%$ and in the fifth, with $10.9 \%$. It was concluded that electroejaculation induced changes in facial expression, vocalization and lying down during the examination. These results suggest the need for refinement of the electroejaculation, improvement of the semen collection protocol, equipment used, facilities and animal handling before, during and after this procedure to improve animal welfare.
\end{abstract}

Keywords: animal welfare; semen collection; behavior; andrological test; bull

\section{Expresión facial, vocalización y posturas anómalas en bovinos sometidos a la eletroejaculación convencional}

RESUMEN. La electroejaculación es utilizada rutinariamente para colectas de semen de los bovinos. Los estudios de las alteraciones de comportamiento en los bovinos sometidos a la electroejaculación convencional son raros, y no se indaga la expresión facial en este procedimiento. Este trabajo tuvo el objetivo de evaluar la expresión facial, vocalización y posturas anómalas en 62 toros sometidos a electroejaculación, en exámenes andrológicos. Se utilizó electroejaculador convencional aplicando, sucesivamente, estímulos eléctricos con intensidades crecientes de corriente continua, hasta cinco estímulos para la obtención de la eyaculación. Las colectas fueron conducidas durante la electroejaculación por las filmaciones de los animales contenidos, enfocando la cabeza y el cuello donde se evaluó la expresión facial, vocalización, posturas de la cabeza, actitud de acostarse, además de la salivación y la eyaculación. La expresión facial fue evaluada por los cambios de comportamiento de la boca y los ojos. En los resultados de los análisis del método de regresión logística hubo efecto significativo al 5\% de probabilidad cuando se consideró la intensidad de los estímulos eléctricos en el procedimiento de la electroejaculación en los registros de las posturas de la cabeza, de los ojos, en la vocalización, en la actitud de acostarse y en la eyaculación. Sin embargo, no hubo efecto significativo cuando se consideró la intensidad de los estímulos eléctricos en el procedimiento de la electroejaculación en los registros de las alteraciones conductuales de la boca y en la ocurrencia de sialorrea. Las posturas de la cabeza fueron $71,0 \%$ en el primer estímulo 
eléctrico; $82,3 \%$ en el segundo estímulo; $85,5 \%$ en el tercero; El 89,7\% en el cuarto y el $82,6 \%$ en el quinto estímulo eléctrico. Los cambios de comportamiento de los ojos presentaron el $74,2 \%$ en el primer estímulo eléctrico; $87,1 \%$ en el segundo y tercer estímulo; El 98,3\% en el cuarto y el 97,8\% en el quinto estímulo eléctrico. Ningún animal acostó durante el primer estímulo eléctrico. La actitud de acostarse en el segundo, tercer, cuarto y quinto estímulos eléctricos fue el 6,5\%; El 4,8\%; El 5,2\% y el 13,0\% respectivamente. Las vocalizaciones fueron más elevadas en el cuarto estímulo eléctrico, con el $12,1 \%$ y el quinto, con el $10,9 \%$. Se concluye que la electroejaculación indujo alteraciones de la expresión facial, vocalización y actitud de acostarse durante el examen. Estos resultados sugieren la necesidad de refinamiento de la electroejaculación, mejoramiento del protocolo de recolección de semen, de los equipos utilizados, de las instalaciones y del manejo de los animales antes, durante y después de este procedimiento para la mejora del bienestar animal.

Palabras clave: Bienestar, recolección de semen, comportamiento, examen andrológico, toro

\section{Introdução}

A contribuição das biotécnicas reprodutivas no desenvolvimento técnico-científico e progresso na produção animal são inegáveis (Figueiredo and Molento, 2008). Segundo estes autores Médicos Veterinários especialistas em biotécnicas reprodutivas fazem parte de uma área emergente e promissora, sendo de responsabilidade deste profissional diagnosticar o impacto destas biotécnicas sobre a qualidade de vidas dos animais, determinando, objetivamente o grau de impedimento de bem-estar, devendo esse grau ser incluído nos processos de tomadas de decisão ética quanto à biotécnicas em questão.

A eletroejaculação é um método utilizado em exames andrológicos de campo para coletas de sêmen para realização do espermiograma, exame necessário para avaliação da fertilidade do touro, bem como para coleta de sêmen para uso comercial nas Centrais de Inseminação Artificial. A coleta de sêmen em bovinos nestas Centrais também pode ser realizada pelo método da vagina artificial ou massagem transretal das glândulas sexuais acessórias, no entanto, estes métodos são limitados e pouco utilizados, sendo às vezes impossível de serem realizados em algumas raças ou indivíduos, os quais não aceitam a realização da eletroejaculação. O método da vagina artificial exige adaptação e condicionamento do touro e da vaca quando utilizada como manequim, enquanto que a eletroejaculação não necessita deste condicionamento. Portanto, a eletroejaculação é o método de eleição utilizado para coletas de sêmen nos exames andrológicos de bovinos criados a campo, não existindo método eficiente para substituição. Na eletroejaculação convencional tem sido observado na prática evidências de alterações comportamentais, sendo raríssimos os estudos relacionados nesta área, não sendo elucidada a expressão facial. Sendo assim, este trabalho teve como objetivo avaliar a expressão facial, vocalização e posturas anômalas em touros submetidos à eletroejaculação convencional, durante os exames andrológicos de rotina.

$\mathrm{Na}$ medicina humana tem sido reconhecido o avanço no conhecimento na ciência do bem-estar. Em Medicina Veterinária este avanço ainda constitui um desafio, notadamente nos animais de produção. Nos procedimentos que causam dor e sofrimento a avaliação do bem-estar animal pode ser realizada por indicadores comportamentais, fisiológicos, hormonais e imunológicos. Devido à impossibilidade de qualquer tipo de verbalização a principal forma de expressar a dor em animais passa a ser por atitudes comportamentais, entre elas a expressão facial, a vocalização e as anormalidades posturais as mais importantes.

A expressão facial é uma das formas de comunicação mais importante nas relações interpessoais em humanos, sendo amplamente utilizada para estudar emoções e a dor, notadamente em pessoas incapazes de comunicação verbal, como recém-nascidos e algumas deficiências que impedem esta comunicação. A face é uma impressão digital de constatação imediata do sofrimento em humanos, desta forma, vem sendo utilizada em diversos estudos, no entanto, nos animais estes estudos são raríssimos, notadamente em animais de produção. Pesquisa liderada pela Universidade McGill, no Canadá, mostra que camundongos também manifestam expressões faciais quando sentem dor. Nesta pesquisa Langford et al. (2010) estudaram a expressão facial em camundongos com objetivo 
de padronizar sistema de código comportamental com alta precisão e confiabilidade, sendo assim, estes autores verificaram a possibilidade de mensurar a dor. Desta forma, inovações científicas neste campo podem ser utilizadas para o desenvolvimento de fármacos que controlam a dor, evitando que animais sofram desnecessariamente.

Nos animais de companhia os estudos relacionados com indicadores comportamentais têm sido utilizados para avaliar o bem-estar, no entanto, em bovinos estes estudos são raríssimos. No Brasil a expressão facial foi estudada na espécie equina por Borja (2008) onde se avaliou a importância dos parâmetros comportamentais e fisiológicos na avaliação clínica mais precisa da dor nesta espécie, entretanto, a expressão facial não foi elucidada na espécie bovina, notadamente durante a eletroejaculação. Sendo assim, a avaliação da expressão facial, vocalização e posturas anormais como indicadores de bem-estar em bovinos têm importância fundamental para exploração econômica e humanitária desta espécie, assim, constituindo um campo promissor de pesquisa, sendo ainda importante para avaliação de protocolos experimentais pelas Comissões de Éticas no Uso de Animais.

\section{Material e Métodos}

Este Projeto foi previamente aprovado pela Comissão de Ética no Uso de Animais do Instituto de Zootecnia de Nova Odessa - SP, Brasil, registrado no Protocolo n ${ }^{\circ} 122$ desta Comissão. O estudo foi conduzido no Centro de Pesquisa de Pecuária de Corte do Instituto de Zootecnia, da Secretaria de Agricultura e Abastecimento (SAA) do Estado de São Paulo - SP, Brasil. Foram analisados os comportamentos de 62 touros durante exames andrológicos de rotina, manejados em sistema de criação extensivo com suplementação mineral e água ad libitum. O estudo do comportamento foi realizado durante o procedimento da eletroejaculação em exames andrológicos de rotina. Após contenção dos animais realizou-se os exames andrológicos, os quais foram compreendidos por exame clínico geral, exame do sistema genital e eletroejaculação, de acordo com o Manual para Exame Andrológico e Avaliação de Sêmen Animal do Colégio Brasileiro de Reprodução Animal. Antes da realização da eletroejaculação procedeu-se a higienização do prepúcio e da região adjacente através da tricotomia, lavagem com água e sabão e antissepsia com álcool a 70\%. A eletroejaculação foi procedida através de estimulação elétrica transretal, utilizando eletroejaculador convencional da marca Tarjet$95 \mathrm{~A}^{\circledR}$ aplicando-se, sucessivamente, estímulos elétricos de correntes crescentes e contínua de 12 volts, de 300 a $500 \mathrm{~mA}$ sendo $300 \mathrm{~mA}$ no primeiro, $350 \mathrm{~mA}$ no segundo, $400 \mathrm{~mA}$ no terceiro, $450 \mathrm{~mA}$ no quarto, $500 \mathrm{~mA}$ no quinto e potência de 4 watts, durante três segundos, intercalados por três segundos de repouso, sendo realizado até cinco estímulos elétricos para promover a ejaculação. $\mathrm{O}$ eletroejaculador utilizado se constituía por central de comando eletro-analógica, sonda retal com eletrodo $(25 \times 8 \mathrm{~cm})$ e fonte de energia, além de cabo de força elétrica.

Os comportamentos dos animais foram registrados por gravação em vídeos, utilizando câmera digital marca Sony DSC-W55, para posterior avaliação. Para tanto, essa câmera foi acoplada a um tripé para estabilização das imagens e facilitar a captação do som para registros da vocalização. A contenção de cada animal foi realizada em tronco convencional, focando principalmente a cabeça e pescoço do animal, no lado esquerdo do tronco de contenção, com a porta lateral anterior aberta para facilitar as filmagens, registros da vocalização e identificação de cada animal, conforme ilustrações das Figuras $1 \mathrm{~A}$ e 1B. As filmagens dos vídeos bem como os registros da vocalização iniciavam-se no instante dos estímulos elétricos da eletroejaculação. A avaliação dos comportamentos foi realizada utilizando-se amostragem focal e coleta contínua das seguintes categorias: expressão facial, vocalização e posturas anômalas.

A expressão facial foi avaliada pelas alterações oculares as quais foram compreendidas por reflexo de abertura de pálpebras e movimentação do globo ocular com exposição da membrana esclerótica e sialorreia (ausente ou presente). $\mathrm{Na}$ vocalização avaliaram-se todos os sons audíveis (gerados na laringe, propagados pelas cavidades ressonantes, incluindo boca e narinas), sendo classificados em breve (duração de até dois segundos) e prolongada (duração acima de dois segundos). As alterações da cabeça foram definidas por movimentações defensivas da cabeça, abaixamento da cabeça, extensão e elevação do pescoço e da cabeça, podendo chegar à posição de opistótono. Avaliou-se ainda a atitude de deitar durante as estimulações elétricas do procedimento da eletroejaculação, conforme ilustrações das Figuras 1C e 1D. 


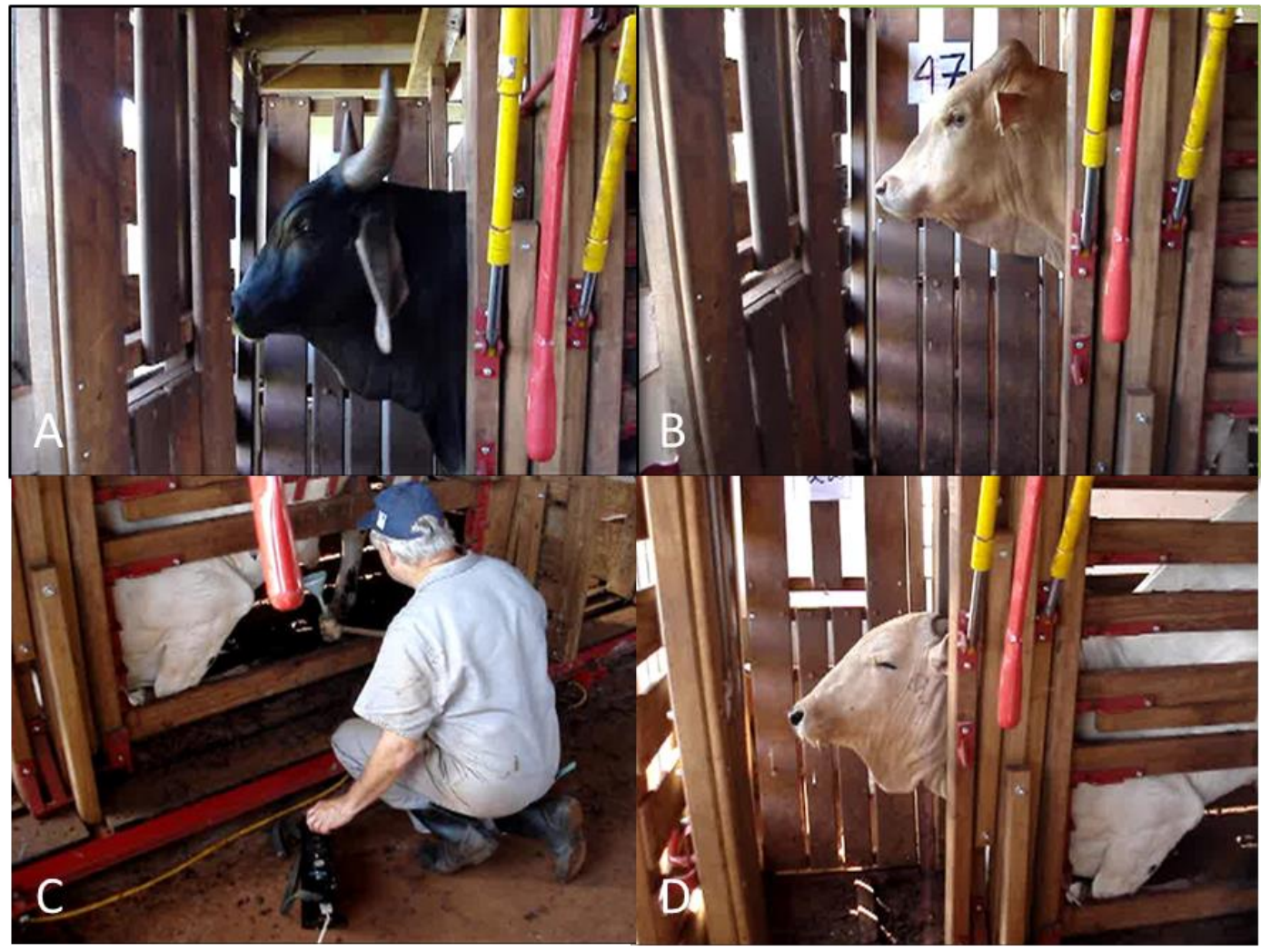

Figura 1. A e B animais contidos sem estimulação elétrica. C. procedimento da eletroejaculação; D animal deitado durante estimulação elétrica transretal.

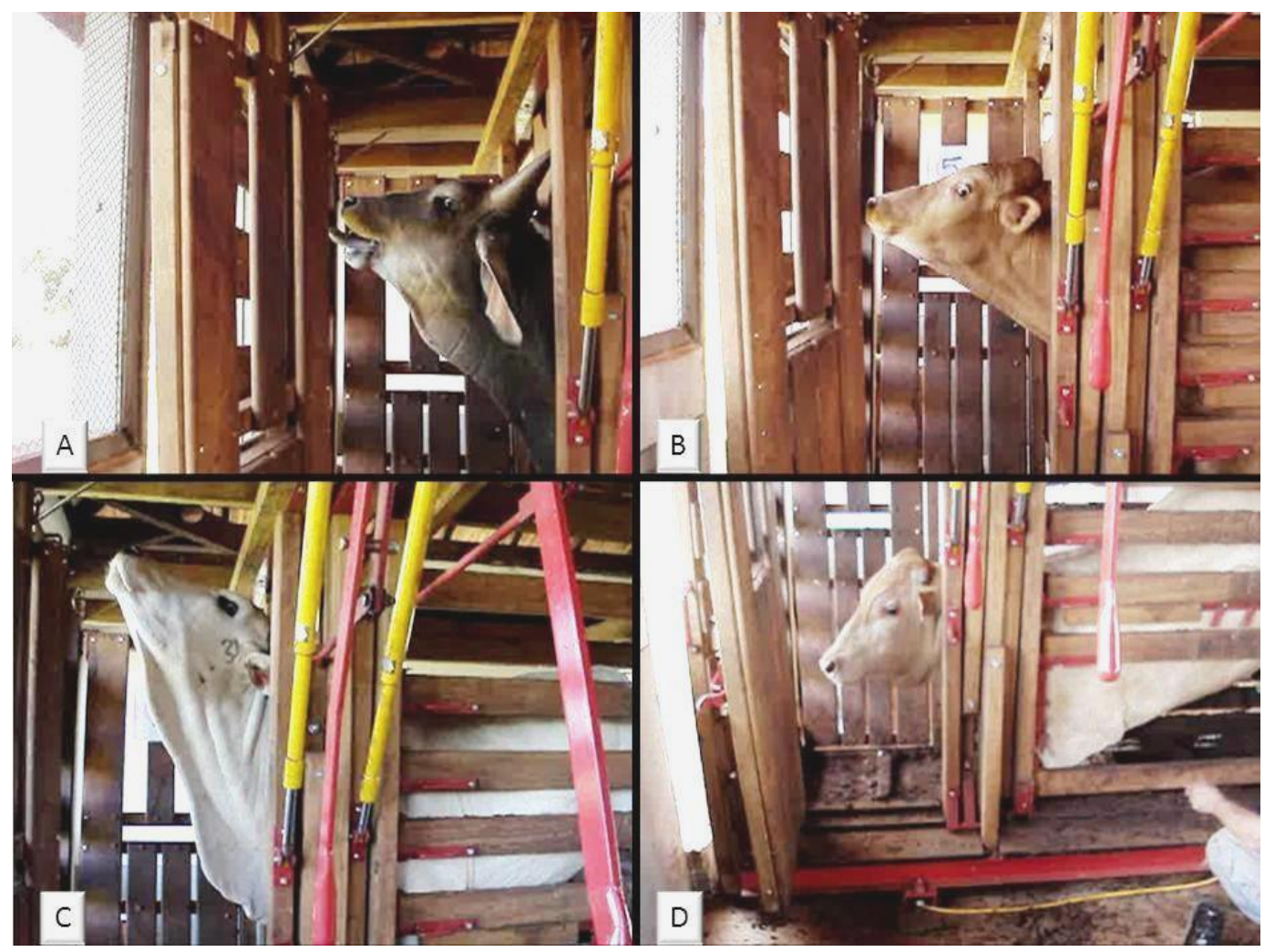

Figura 2. Behavioral changes in facial expression, vocalization and lying down during the procedure of electroejaculation: A - Vocalization and mouth opening with tongue protrusion, B - Ocular alteration and neck extension; C - Elevation of the head in opisthotonos position; D - Lying down and evidence of sialorrhea. 
Análises estatísticas. As respostas comportamentais dos animais nas intensidades de estímulos elétricos são binárias, com valores 0 , se não tiver resposta e 1 se tiverem resposta. As intensidades de estímulos, medidos em mA, são quantitativos e contínuos. A relação entre a variável de resposta (comportamento animal) e a variável preditor (intensidade de estímulo em $\mathrm{mA}$ ) foi analisada por método de regressão logística binária, usando o logit da função de link, como o modelo:

$$
g\left(\pi_{j}\right)=\beta_{0}+\beta \mathbf{x}_{j}^{\prime}
$$

Onde:

$\pi_{j}=$ A probabilidade de uma resposta para o $j^{\text {th }}$ padrão de fatores.

Link logito função, $g\left(\pi_{j}\right)=\log _{e}\left(\frac{\pi_{j}}{1-\pi_{j}}\right)$

$\beta_{0}=\mathrm{A}$ intercepção

$x_{j}^{\prime}=$ Um vetor de variável preditor associado ao $j^{\text {th }}$ padrão de fatores

$\beta=\mathrm{Um}$ vetor de coeficientes desconhecidos associados ao preditor.

O modelo ajustou-se usando um algoritmo de mínimos quadrados com pontuação interativa para obter estimativas de máxima verossimilhança de parâmetros. A hipótese nula $H_{0}: \beta=0$ e hipótese alternative $H_{a}: \beta \neq 0$ foi testada com nível $\propto>$ 0.05 . As análises foram realizadas com o Minitab, versão 13.

\section{Resultados}

A Tabela 1 apresenta os resultados da análise de regressão logística, com modelo logito, para predizer o logito das variáveis dicotômicas de regressão de comportamento (Y) para a intensidade de estímulo elétrico (X). O logito é a proporção (odds) de $\mathrm{Y}=1$ (apresentar $\mathrm{o}$ comportamento). De acordo com os resultados das análises do modelo de regressão logístico ajustado (Tabela 1) não houve efeito significativo quando se considerou a intensidade dos estímulos elétricos no procedimento da eletroejaculação nos registros das alterações comportamentais da boca, incluindo abertura da boca com protrusão de língua e sem protusão de língua (Test $\mathrm{Z}, \mathrm{p}=$ 0.595). Não é rejeitada a hipótese de nulidade de $\beta=0$, portanto, não há evidência que estas intensidades de estímulos elétricos estimulem alterações comportamentais da boca. Nesta variável, quando se considerou os estímulos elétricos aplicados em todos os animais ocorreram apenas $1,72 \%$ de alterações comportamentais. A maior frequência de resposta de abertura da boca ocorreu no quarto estímulo elétrico, com porcentagem de $3,45 \%$.

Os estímulos de correntes elétricas induziram alterações comportamentais da cabeça, incluindo as movimentações defensivas da cabeça, abaixamento da cabeça, extensão e elevação do pescoço e da cabeça, podendo chegar à posição de opistótono, houve efeito significativo $(\mathrm{p}<0.05)$. Para cada aumento em unidade de intensidade de corrente elétrica há um aumento no $\log$ de $\mathrm{P}$ (manifestar comportamento, $\mathrm{Y}=1$ ) / $\mathrm{P}$ (não manifestar comportamento, $\mathrm{Y}=0$ ) igual a 0.004732 (Tabela 1). Portanto, há evidências de que os estímulos elétricos utilizados no procedimento influenciem a variável alteração comportamental da cabeça. Quando se considerou todos os estímulos elétricos observou-se que ocorreram $82,07 \%$ de alterações comportamentais da cabeça, sendo $70,97 \%$ para o primeiro estímulo; $82,26 \%$ para o segundo estímulo; $85,48 \%$ para o terceiro estímulo; $89,66 \%$ para o quarto estímulo e $82,61 \%$ para o quinto estímulo elétrico.

Ao analisar o comportamento da atitude de deitar, durante as estimulações elétricas da eletroejaculação, verificou-se que houve efeito significativo $(p<0.05)$. De forma semelhante, houve evidências que o aumento da intensidade da corrente elétrica aplicada no procedimento avaliado neste trabalho influenciou no aumento da atitude de deitar. Quando se considerou todos os estímulos elétricos verificou-se uma porcentagem de $5,52 \%$, sendo observada a maior porcentagem no quinto estímulo elétrico, igual a $13,04 \%$.

De forma semelhante, quando se avaliou a ocorrência de ejaculação, nas estimulações de correntes elétricas utilizadas, verificou-se que houve efeito significativo ( $\mathrm{p}<0.05)$. Desta forma, também houve indicação de que a aplicação de intensidades mais elevadas de estímulos de correntes elétricas usadas neste trabalho estimule a ejaculação. Do total de observações analisadas verificou-se, nos 62 animais avaliados, uma porcentagem de ejaculação $6,45 \%$ no terceiro estímulo elétrico; em 58 animais analisados no quarto estímulo elétrico uma porcentagem de $20,69 \%$ e em 46 animais avaliados no quinto estímulo elétrico uma porcentagem de $41,30 \%$, sendo a taxa total de ejaculação de 56,45\%. 
As descargas de correntes elétricas utilizadas na eletroejaculação influenciaram também alterações comportamentais dos olhos, incluindo os reflexos de abertura de pálpebras e as movimentações do globo ocular com exposição da membrana esclerótica, tendo efeito significativo $(\mathrm{p}<0.05)$. Portanto, houve evidências que o procedimento da eletroejaculação com intensidades crescentes de estímulos elétricos estimule alterações comportamentais dos olhos. Do total de observações analisadas houve $88,28 \%$ de alterações neste comportamento, aumentando gradativamente de acordo com o aumento da intensidade do estímulo elétrico aplicado, sendo $74,19 \%$ no primeiro estímulo e $87,10 \%$ no segundo e terceiro estímulo elétrico aplicados em 62 animais. Analisando ainda este comportamento houve $98,28 \%$ de alterações no quarto estímulo em 58 animais avaliados e 97,83 de alterações deste comportamento em 46 animais avaliados.

A variável ocorrência de salivação foi considerada quando presente ou ausente. Ao analisar a resposta comportamental com esta variável verificou-se que não houve efeito significativo ( $\mathrm{p}=0,124)$, não havendo, portanto, efeito do aumento dos estímulos elétricos na salivação. A porcentagem de animais que manifestaram este comportamento foi de $22,41 \%$ quando se avaliou o total de observações.

A vocalização foi estudada quando associada ou não a abertura de boca, com ou sem protusão da língua, onde se avaliou os sons audíveis (gerados na laringe, propagados pelas cavidades ressonantes, incluindo boca e narinas), sendo classificados em breve (duração de até dois segundos) e prolongada (duração acima de dois segundos). A intensidade de correntes elétricas aplicadas na eletroejaculação teve efeito significativo na ocorrência da vocalização, $(\mathrm{p}<0.05)$, observado na Tabela 1 . Ao analisar esta variável verificou-se na Tabela 1 efeito significativo $(\mathrm{p}<0.05)$. Portanto, há evidências que o aumento nas intensidades de correntes elétricas aplicada estimule a vocalização. No total de observações verificou-se uma porcentagem de $5,52 \%$, sendo mais elevada no quarto e no quinto estímulo elétrico, com $12,07 \%$ e $10,87 \%$ respectivamente (Tabela 2$)$.

Tabela 1. Logística, regressão, modelo logit do comportamento e variáveis elétricas

\begin{tabular}{|c|c|c|c|c|c|c|}
\hline Variáveis de comportamento & Preditor & Coeficiente & SE Coef & $\mathrm{Z}$ & $\mathrm{P}$ & Odds $(95 \% \mathrm{CI})$ \\
\hline \multirow{2}{*}{ Alterações da boca } & Constante $\alpha$ & $-5,447 *$ & 2,744 & $-1,99$ & 0,047 & \\
\hline & Corrente $\beta$ & 0,003496 n.s & 0,006607 & 0,53 & 0,597 & $1,0035(0.99,1.02)$ \\
\hline \multirow{2}{*}{ Alterações da cabeça } & Constante $\alpha$ & $-0,3089$ n.s & 0,891 & $-0,35$ & 0,729 & \\
\hline & Corrente $\beta$ & $0,004732 *$ & 0,002311 & 2,05 & 0,041 & $1,0047(1.00,1.01)$ \\
\hline \multirow{2}{*}{ Atitude de deitar } & Constante $\alpha$ & $-6,806^{*}$ & 1,793 & $-3,80$ & 0,000 & \\
\hline & Corrente $\beta$ & $0,00959 *$ & 0,004106 & 2,34 & 0,020 & $1,0096(1.00,1.02)$ \\
\hline \multirow{2}{*}{ Ejaculação } & Constante $\alpha$ & $-13,71 *$ & 2,168 & $-6,32$ & 0,000 & \\
\hline & Corrente $\beta$ & $0,027004 *$ & 0,004666 & 5,79 & 0,000 & $1,0273(1.02,1.04)$ \\
\hline \multirow{2}{*}{ Alterações dos olhos } & Constante $\alpha$ & $-3,066^{*}$ & 1,2 & $-2,55$ & 0,011 & \\
\hline & Corrente $\beta$ & $0,01371 *$ & 0,003378 & 4,06 & 0,000 & $1,0138(1.01,1.02)$ \\
\hline \multirow{2}{*}{ Sialorreia } & Constante $\alpha$ & $-2,5038 *$ & 0,8409 & $-2,98$ & 0,003 & \\
\hline & Corrente $\beta$ & 0,003172 n.s. & 0,002062 & 1,54 & 0,124 & $1,0032(1.00,1.01)$ \\
\hline \multirow{2}{*}{ Vocalização } & Constante $\alpha$ & $-7,978 *$ & 1,945 & $-4,10$ & 0,000 & \\
\hline & Corrente $\beta$ & $0,012275^{*}$ & 0,004371 & 2,81 & 0,005 & $1,0123(1.00,1.02)$ \\
\hline
\end{tabular}

*Coeficientes significativos a $5 \%$ de probabilidade, pelo teste Z, Coeficientes não significativos (n.s) a 5\% de probabilidade, pelo teste Z.

$\mathrm{O}$ odds rate indica o aumento esperado da porcentagem no odds de manifestar a resposta comportamental quando se aumenta a intensidade de corrente elétrica em uma unidade. Assim, considerando que as intensidades de correntes elétricas têm uma amplitude de $50 \mathrm{~mA}$, quando altera a intensidade de estímulo, o aumento na porcentagem esperada da probabilidade de manifestar as respostas comportamentais é igual a: alterações comportamentais da cabeça $(23,7 \%)$, atitude de deitar $(48,2 \%)$, ejaculação $(136,9 \%)$, alterações comportamentais dos olhos $(69,0 \%)$ e vocalização $(61,8 \%)$. 
Tabela 2. Percentagem de respostas comportamentais em relação à quantidade de animais submetidos às intensidades de estímulos elétricos

\begin{tabular}{lcccccccc}
\hline $\begin{array}{l}\text { Estímulo } \\
\text { Elétrico }\end{array}$ & $\begin{array}{c}\text { No }^{\text {de }} \\
\text { Animais }\end{array}$ & $\begin{array}{c}\text { Alterações } \\
\text { Boca }\end{array}$ & $\begin{array}{c}\text { Alterações } \\
\text { Cabeça }\end{array}$ & $\begin{array}{c}\text { Atitude } \\
\text { Deitar }\end{array}$ & $\begin{array}{c}\text { Ocorrência } \\
\text { Ejaculação }\end{array}$ & $\begin{array}{c}\text { Alterações } \\
\text { Oculares }\end{array}$ & $\begin{array}{c}\text { Ocorrência } \\
\text { Sialorreia }\end{array}$ & Vocalização \\
\hline 1 & 62 & $1,61 \%$ & $70,97 \%$ & $0,00 \%$ & $0,00 \%$ & $74,19 \%$ & $17,74 \%$ & $1,61 \%$ \\
2 & 62 & $1,61 \%$ & $82,26 \%$ & $6,45 \%$ & $0,00 \%$ & $87,10 \%$ & $17,74 \%$ & $1,61 \%$ \\
3 & 62 & $0,00 \%$ & $85,48 \%$ & $4,84 \%$ & $6,45 \%$ & $87,10 \%$ & $25,81 \%$ & $3,23 \%$ \\
4 & 58 & $3,45 \%$ & $89,66 \%$ & $5,17 \%$ & $20,69 \%$ & $98,28 \%$ & $24,14 \%$ & $12,07 \%$ \\
5 & 46 & $2,17 \%$ & $82,61 \%$ & $13,04 \%$ & $41,30 \%$ & $97,83 \%$ & $28,26 \%$ & $10,87 \%$ \\
\hline Total & 290 & $1,72 \%$ & $82,07 \%$ & $5,52 \%$ & $12,07 \%$ & $88,28 \%$ & $22,41 \%$ & $5,52 \%$ \\
\hline
\end{tabular}

\section{Discussão}

No presente trabalho verificou-se que as intensidades de correntes elétricas aplicadas durante a eletroejaculação nos bovinos não causaram alterações comportamentais na boca. Portanto, esta variável não mostrou importância na avaliação do comportamento dos animais submetidos este procedimento. Contudo, nem toda alteração da expressão facial pode ser visualizada pelo observador, podendo ocorrer movimentos imperceptíveis. Algumas expressões faciais são muito claras e revela prontamente o sentimento de um indivíduo, outras são difíceis de serem evidenciadas ou interpretadas. Desta forma, o mapeamento dos traços das alterações comportamentais da expressão facial abre um campo promissor de estudos mais detalhados para avaliação do bem-estar de animais de produção, os quais são frequentemente submetidos a procedimentos de rotina, que aparentemente não interferem na qualidade de vida dos mesmos. Portanto, a face pode ser uma impressão digital de constatação imediata do sofrimento animal.

Quando se avaliou as alterações comportamentais da cabeça verificou-se que as intensidades de correntes elétricas aplicadas durante a eletroejaculação influenciaram nesta variável de comportamento. Na Figura 3 mostra que a probabilidade de expressar este comportamento aumenta com a intensidade dos estímulos elétricos aplicados durante este procedimento. Provavelmente este comportamento seja desencadeado como mecanismo de defesa em decorrência do estresse, da contenção e do desconforto. Esta evidência está de acordo com Broom and Molento (2004) os quais enfatizaram que quanto mais forte as reações de defesas nos animais, mais pobre será o bemestar durante um estímulo aversivo. Segundo estes autores um indivíduo que se encontra impossibilitado de adotar uma postura preferida de conforto, ou de repouso, será considerado como um indivíduo que tem o bem-estar mais pobre que outro cuja situação permite a adoção da postura preferida. Comportamento semelhante a este foi observado na Tabela 2 no presente trabalho, onde houve uma porcentagem de $82,1 \%$ de alterações comportamentais da cabeça, em decorrência das estimulações elétricas da eletroejaculação. De forma semelhante, os resultados do presente trabalho sugerem importante padrão comportamental que revelou comprometimento do bem-estar animal, podendo ser caracterizado por desconforto, frustração e estresse, não descartando a possibilidade de sofrimento.

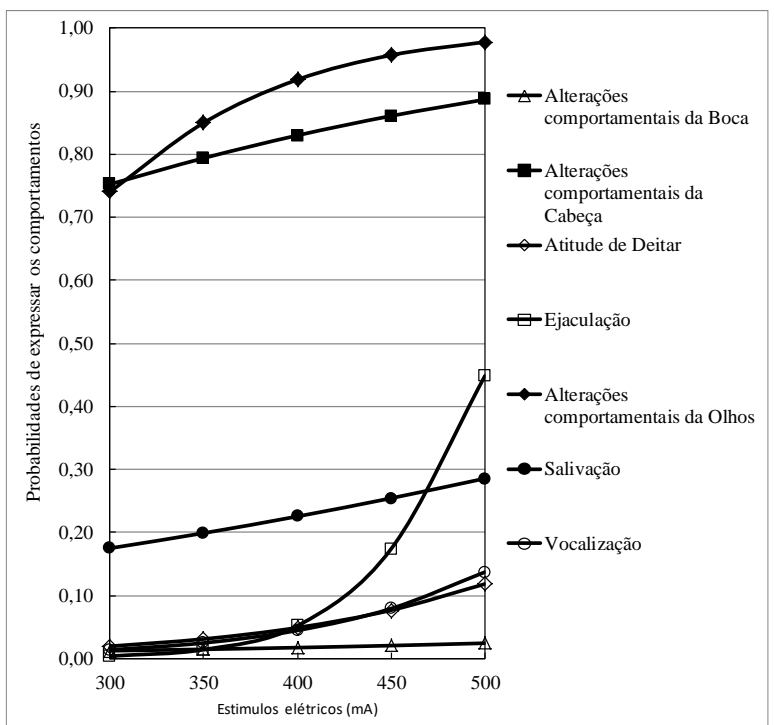

Figura 3. Resultados da ocorrência das variáveis comportamentais de acordo com a intensidade dos estímulos elétricos durante o procedimento da eletroejaculação.

Nas Figuras 2/B e 2/C ilustram exemplos destas alterações comportamentais, as quais evidenciam extensão e elevação da cabeça e pescoço, até a posição de opistótono. Especula-se que as alterações comportamentais da cabeça não sejam específicas do procedimento da eletroejaculação, podendo está relacionado a outras condições que causam estresse, desconforto, dor, entre outras condições. Portanto, 
entender melhor este comportamento auxilia o Médico Veterinário na intervenção de forma que possa evitar situações de estresse, desconforto ou até distúrbios psicológicos, que possam comprometer o bem-estar dos animais submetidos a este procedimento.

No presente estudo houve evidências que o aumento da intensidade de corrente elétrica aplicada durante $\mathrm{o}$ procedimento de eletroejaculação influenciou no aumento da atitude de deitar, sendo este comportamento considerado aversivo ao procedimento, levando ainda em consideração a contenção do pescoço e de todo corpo pelas guilhotinas do tronco de contenção, impossibilitando os movimentos de liberdade, condição física que deveria dificultar o comportamento da atitude de deitar durante a aplicação dos estímulos elétricos do procedimento. $\mathrm{O}$ gráfico evidenciado na Figura 3 mostra que a probabilidade de o animal expressar este comportamento aumenta com a intensidade dos estímulos elétricos aplicados. Quando se considerou todos os estímulos elétricos verificouse que nenhum animal deitou durante o primeiro estímulo, indicando que este não foi suficiente para causar estresse ou desconforto. O estímulo elétrico de maior intensidade correspondeu com a maior porcentagem de ocorrência de atitude de deitar (13,0\%), denunciando estresse e desconforto dos animais durante o exame. Nas condições em que o presente trabalho foi realizado verificou-se que o protocolo utilizado foi suficiente para causar desconforto e estresse, notadamente quando se aplicou maior intensidade de corrente elétrica, conforme ilustrações das Figuras 1/D e 2/D do presente trabalho, as quais evidenciam ilustrações da atitude de deitar durante as estimulações elétricas transretais, ocorridas entre o terceiro e quinto estímulos. Especula-se que exista variação quanto a este comportamento em decorrência do manejo geral e manejo de contenção aplicado, tipo de tronco de contenção, modelo do eletroejaculador e do protocolo de eletroejaculação aplicado. Atualmente a indústria vem fabricando diversos tipos de troncos de contenção animal, sendo que alguns impedem que o animal deite durante procedimento de eletroejaculação. No estudo realizado por Etson et al. (2004) foi observado que nenhum animal deitou quando se utilizou protocolos com sonda retal convencional e segmentada, aplicando ou não anestesia epidural caudal. Entretanto, estes autores enfatizaram que touros jovens deitam, vocalizam ou se debatem, quando submetidos a intensidades elevadas de correntes elétricas. O trabalho conduzido por Falk et al. (2001) mostrou que $38,70 \%$ dos animais deitaram durante o procedimento de eletroejaculação convencional. No entanto, quando estes autores utilizaram o protocolo com anestesia epidural caudal, apenas $22,6 \%$ dos animais deitaram durante este procedimento. Marques Filho et al. (2008) verificaram maiores valores de frequência da atitude de deitar durante a eletroejaculação, sugerindo que a estimulação elétrica transretal foi suficientemente desagradável para desencadear a atitude de deitar. As diferenças nas respostas de atitude de deitar é um indicativo de que o aprimoramento da técnica de eletroejaculação, bem como boas práticas de manejo e instalações adequadas possam contribuir com o bem-estar dos animais.

$\mathrm{Na}$ Tabela 1 do presente trabalho mostra a ocorrência de ejaculação e verifica-se que teve efeito significativo $(p<0.05)$. Como era de se esperar a aplicação do procedimento da eletroejaculação pelo método convencional promove a ejaculação. A Tabela 2 e a Figura 3 mostram que no primeiro e segundo estímulo de corrente elétrica nenhum animal ejaculou, embora já manifestassem sinais de desconforto e estresse, de modo crescente. A taxa de ejaculação dos animais foi de 56,5\%. Este resultado é um indicativo de que o procedimento da eletroejaculação funcionou, sendo esta necessária para coletas de sêmen para realização do espermiograma, no entanto, não significa que a mesma não comprometeu o bem-estar dos animais. Verificou que no primeiro e seguindo estímulo de corrente elétrica nenhum animal ejaculou, embora já manifestassem sinais de estresse e desconforto, os quais foram crescentes, à medida que aumentasse a intensidade de estimulações elétricas transretais. Estes resultados foram semelhantes aos encontrados por Marques Filho et al. (2008) no qual obtiveram porcentagem de $66,7 \%$ na taxa de ejaculação. No estudo realizado por Falk et al. (2001) foi obtido uma taxa de ejaculação de $93,1 \%$, enquanto que Etson et al. (2004) evidenciaram $100 \%$ nesta taxa. Independente da taxa de ejaculação obtida nos diversos trabalhos da literatura observa-se que existe uma variação nas porcentagens, sendo estas dependentes do protocolo utilizado, do tipo de equipamento, do manejo dos animais, bem como do operador. Alguns trabalhos têm sido realizados com objetivo de testar protocolos de eletroejaculação utilizando fármacos para o 
aprimoramento e refinamento da técnica de eletroejaculação, com objetivo de promover o bem-estar dos animais. Como exemplo no estudo realizado por Falk et al. (2001), onde foi avaliado os efeitos da administração de lidocaína por via epidural caudal. Os autores deste trabalho verificaram os benefícios do uso de fármacos utilizados para amenizar os efeitos do estresse desencadeado pela técnica, sem interferência na taxa de ejaculação. Portanto, destaca-se a importância do presente trabalho o qual mostra os efeitos da técnica de estimulação elétrica transretal da eletroejaculação nos exames de rotina no comportamento dos animais, notadamente da expressão facial.

O procedimento da eletroejaculação provocou também alterações comportamentais nos olhos, de forma semelhante percebe-se na Figura 3 que a probabilidade deste comportamento ser evidenciado aumenta com o aumento da intensidade dos estímulos elétricos. A porcentagem de animais que apresentaram este comportamento foi de $88,28 \%$. De forma similar estas alterações comportamentais aumentaram gradativamente, de acordo com a intensidade de corrente elétrica aplicada, sendo 74,2 no primeiro estímulo elétrico e 97,2 no quinto. Estas alterações evidenciam importante indicador de estresse, frustração, descontentamento ou temperamento. No entanto, não devem ser consideradas específicas do procedimento da eletroejaculação, podendo ocorrer em procedimentos que afetam a emoção e sofrimento, comprometendo o bemestar, como mostram os trabalhos realizados por Sandem et al. (2002), Sandem and Braastad (2005), Sandem et al. (2006), Core et al. (2009) os quais demonstram que estas alterações ocorrem em condições de estresse. Sendo assim, os resultados do presente trabalho mostram que as alterações oculares representam excelentes indicadores comportamentais para avaliação do bem-estar em bovinos, o que estar de acordo Broom (2006) os quais citam alterações oculares com movimentação dos olhos em animais submetidos a condições que afetam o bem-estar. Segundo Paranhos da Costa (2004) o temperamento é definido como um conjunto de traços psicofisiológicos estáveis de um dado indivíduo, determinando suas reações emocionais. De acordo com Diesel et al (2011) o temperamento está relacionado com características produtivas e facilidade de manejo dos animais, no entanto, é de fácil mensuração, tornando um desafio à definição de metodologias que permitam uma avaliação eficiente e prática. Ao estudar o comportamento de exposição da membrana esclerótica como indicador do temperamento em bovinos, durante os procedimentos de manejo, estes autores concluíram que não é um bom indicador comportamental. Entretanto, avaliaram apenas animais durante manejo de pesagem, o qual não é considerado invasivo, capaz de causar dor e sofrimento animal, talvez por não constituir estímulo suficiente para alterar o comportamento com evidência de alterações oculares. No presente estudo avaliou-se a eletroejaculação, que é considerada como um procedimento invasivo, o qual se aplica estímulos elétricos de correntes elétricas de intensidades crescentes, capazes de induzir expressivas alterações comportamentais, envolvendo a expressão facial, vocalização e posturas anômalas, conforme ilustrações das Figuras 1/D e 2/A,B,C e D.

A porcentagem da exposição da membrana esclerótica em bovinos leiteiros foi estudada por Sandem and Braastad (2005) como indicadora de frustração. Estes autores mostraram que esta alteração ocular apresentou maior porcentagem no período de separação dos bezerros das matrizes. Estes autores reforçam que esta alteração comportamental representa um importante indicador de emoções. Em outro trabalho a exposição da membrana esclerótica foi estudada foi estudada por Sandem et al. (2006). Ao avaliar este comportamento em bovinos leiteiros submetidos a condições de estresse ou não estes autores verificaram que os animais com estresse foram mais afetados por esta alteração ocular. Quando utilizaram o ansiolítico diazepam no controle das emoções, em dois grupos de bovinos, verificaram que os animais tratados com este fármaco apresentaram menor porcentagem de exposição da membrana esclerótica, concluindo que esta alteração ocular tem importância na avaliação da emoção nesta espécie. Este comportamento tem sido utilizado também para estudar o temperamento em bovinos de corte. Com objetivo de estudar a ocorrência da exposição da membrana esclerótica como indicador do temperamento. Core et al (2009) mostraram este comportamento pode ser utilizado como ferramenta quantitativa para a seleção de bovinos de corte, podendo assim ser utilizada para avaliar o temperamento em bovinos de corte.

Não houve efeito significativo do aumento da intensidade de corrente elétrica na ocorrência ou não da salivação. Quando se avaliou o total de 
observações à porcentagem de animais que manifestaram este comportamento foi de $22,4 \%$ (Tabela 2). No entanto, alguns trabalhos tem mostrado que este indicador foi considerado importante na avaliação do bem-estar animal, incluindo aqueles relacionados ao procedimento da eletroejaculação. Segundo Bassanezi and Oliveira Filho (2006) o reconhecimento da dor em humanos é avaliado através da somatória de parâmetros fisiológicos que consiste na demonstração de sinais como sialorreia, frequência cardíaca, frequência respiratória, vocalização e avaliação endócrina. Nos estudos realizados por Marques Filho et al. (2008) evidenciaram que os estímulos elétricos do eletroejaculador causaram sialorreia, sendo relacionada ao estresse. De acordo com Goodbourn et al. (2000) a sialorreia provavelmente reflete uma maior atividade das glândulas salivares devido à estimulação parassimpática. Entretanto, Mosure et al. (1998) realizaram um estudo com objetivo de amenizar a dor durante a eletroejaculação e evidenciaram que os animais apresentaram salivação copiosa, no entanto, a mesma estava relacionada aos efeitos do cloridrato de xilazina utilizada no protocolo farmacológico do experimento.

Houve evidências que o aumento nas intensidades de correntes elétricas aplicada estimule a vocalização. No total de observações verificou-se uma porcentagem de 5,5\%, aumentando proporcionalmente com a intensidade de corrente elétrica, sendo mais elevado no quarto e quinto estímulo elétrico, com $12,1 \%$ e $10,9 \%$, respectivamente (Tabela 2 ), havendo assim, uma elevação considerável em relação ao primeiro estímulo, com 1,6\%. Este resultado mostra com o aumenta da intensidade de corrente elétrica pode comprometer o bem-estar animal, entretanto, a ausência da vocalização não significa que o bemestar não esteja comprometido. Na Figura 3 pode ser evidenciado também que probabilidade para este comportamento ser expresso aumenta com a intensidade dos estímulos elétricos aplicados. Sendo assim, os registros da vocalização mostraram ser eficazes, facilitando a identificação dos animais submetidos a condições de estresse e desconforto, durante a eletroejaculação.

Segundo Amyx (1987) uma das características da dor ou angústia nos animais está relacionada com mudança no comportamento e na manifestação de reflexo. No entanto, segundo Muir and Gaynor (2009) apesar do fato do animal demonstrar comportamento que possa estar relacionado à dor não significa que o mesmo esteja sentindo dor. Entretanto, segundo Watts and Stookey (2000) a vocalização é um ótimo indicador comportamental para avaliar a dor, e seus graus são importantes para mensurá-la quando associada à eletroejaculação em touros (Palmer, 2005). No trabalho realizado por Falk et al. (2001) foi evidenciado a ocorrência de $25,8 \%$ de vocalização em animais submetidos a eletroejaculação convencional, sendo que nenhum animal vocalizou de forma discreta e $12,9 \%$ de forma moderada e severa. Estes autores observaram que quando os animais foram submetidos à anestesia epidural caudal apenas $6,5 \%$ vocalizaram de forma discreta. Marques Filho et al. (2008) evidenciaram aumento na frequência de vocalização em bovinos submetidos a eletroejaculação quando comparados ao grupo controle, ou seja, sem submissão a este procedimento. Em um estudo realizado por Whitlock et al. (2012) foi mostrado aumento significativo da ocorrência de vocalização, além dos níveis plasmáticos de progesterona e cortisol, os quais, provavelmente, estavam associados a estresse agudo em touros após o procedimento da eletroejaculação. Entretanto, Etson et al. (2004) utilizaram um sistema subjetivo de pontuação para avaliar o comportamento de touros submetidos a diferentes métodos de eletroejaculação e verificaram que nenhum animal vocalizou quando submetidos a eletroejaculação sob aplicação da anestesia epidural caudal ou sem aplicação desta anestesia. A palpação direta ou manipulação de regiões sensíveis usualmente induz uma resposta, como, vocalização, escape, fuga, retirada, ou coice (Muir and Gaynor, 2009). De acordo com estes autores a avaliação destes aspectos comportamentais relacionados à dor demanda mais tempo, mas resulta numa melhor avaliação de diferentes condições dolorosas não determinadas.

A literatura mostra, de forma clara, que a vocalização em animais é um indicador de estresse e dor e que constitui um campo promissor de pesquisa a ser explorado. $\mathrm{O}$ presente trabalho mostrou que a porcentagem de animais que vocalizaram no penúltimo e último estímulo elétrico, quarto estímulo $(12,1 \%)$ e quinto estímulo $(10,9 \%)$ respectivamente, os quais estão relacionados com o aumento da intensidade de corrente elétrica devido ao desconforto, estresse ou até sensações dolorosas, comparado com o primeiro e segundo estímulos elétricos $(1,61 \%)$. Salienta-se que o conceito de dor é complexo, 
difícil de ser definido, tanto em relação a sua extensão, como suas consequências e nem sempre está associada a danos teciduais (Muir and Gaynor, 2009). A definição mais aceita foi descrita pela International Association for the Study of Pain que define a dor como sendo uma experiência emocional e sensitiva desagradável associada a uma lesão real ou potencial de um tecido. No presente trabalho não se descarta a presença ou não da dor, existe dificuldade na sua avaliação pelo fato da mesma ser uma experiência subjetiva, portanto, não é possível provar sua presença, mas pode-se confirmar a existência de sinais associados a condições dolorosas. Assim, no presente trabalho as respostas comportamentais da expressão facial, os movimentos corporais e a vocalização fornecem informações sobre o estresse e desconforto, as quais denunciam um procedimento doloroso.

As características posturais dos animais estão entre os mais comuns aspectos comportamentais que se alteram nas doenças, condições mecânicas, nervosas, de alterações adaptativas permanentes ou condições de dor (Broom, 2006). De forma semelhante, as alterações posturais e sensoriais ocorrem durante procedimentos de rotina ou em condições experimentais, entre elas a eletroejaculação, aspiração folicular, inseminação artificial, transferência de embriões, castração, descorna, marcação com ferro quente, entre outras. Sendo assim, faz-se necessário a realização de estudos que mostrem o grau de comprometimento necessário para avaliar estresse, dor e sofrimento dos animais, importante para aplicação de métodos alternativos e de refinamento nos sistemas de produção dos bovinos.

\section{Conclusão}

Fundamentada nas condições em que este estudo foi realizado conclui-se que a metodologia desenvolvida foi adequada, podendo ser utilizada em inovações científicas para serem aplicadas em outros trabalhos. A avaliação da expressão facial, da vocalização e a atitude de deitar durante a eletroejaculação teve importância fundamental, sinalizando estresse, desconforto e sofrimento, não descartando a possibilidade da ocorrência de sensações dolorosas. Estas alterações comportamentais revelam que a estimulação elétrica transretal foi suficientemente desagradável e contribuem para o monitoramento do grau de bem-estar animal, mostram ainda a necessidade de aprimoramento da técnica de eletroejaculação, bem como do aperfeiçoamento do eletroejaculador. Desta forma, os resultados comprovam a importância de estudos direcionados ao refinamento desta técnica, através de métodos alternativos à eletroejaculação convencional; pela utilização ou desenvolvimento de modelos de eletroejaculadores mais eficientes; pela realização de manejo humanitário dos animais, antes, durante e após a eletroejaculação e, principalmente, o desenvolvimento de protocolos, utilizando-se analgesia multimodal, a qual consiste no uso de múltiplas drogas, com diferentes ações, que podem agir em diferentes níveis das vias nociceptivas, para produzir uma analgesia ideal. Desta forma, estes métodos alternativos podem minimizar o sofrimento animal, e consequentemente melhorar a qualidade de vida dos mesmos submetidos a este procedimento, principalmente aqueles doadores de sêmen, os quais são utilizados rotineiramente nas centrais de inseminação artificial.

\section{Referências Bibliográficas}

Amyx, H. L. 1987. Control of animal pain and distress in antibody production and infectious disease studies. Journal of the American Veterinary Medical Association, 191, 12871289.

Bassanezi, B. S. B. \& Oliveira Filho, A. G. 2006. Analgesia pós-operatória. Revista do Colégio Brasileiro de Cirurgiões, 33, 116-122.

Borja, M. C. 2008. Avaliação da dor no pósoperatório de artroscopia em equinos. [Dissertação], São Paulo: Faculdade de Medicina Veterinária e Zootecnia, Universidade de São Paulo.

Broom, D. M. 2006. Behaviour and welfare in relation to pathology. Applied Animal Behaviour Science, 97, 73-83.

Broom, D. M. \& Molento, C. F. M. 2004. Animal welfare: concept and related issues-review. Archives of Veterinary Science, 9, 1-11.

Core, S., Widowski, T., Mason, G. \& Miller, S. 2009. Eye white percentage as a predictor of temperament in beef cattle. Journal of Animal Science, 87, 2168-2174.

Diesel, T. A.; Paço, A. L.; Cruz, V. A. R.; Silva, M. L. P.; Tullio, R. R.; Alencar, M. M. A 2011. Exposição da membrana esclerótica como indicativo do temperamento em bovinos. In: Reunião Anual da Sociedade Brasileira de Zootecnia, 48, Belém. Anais... Belém: SBZ, 2011. (CD ROM). 
Etson, C. J., Waldner, C. L. \& Barth, A. D. 2004. Evaluation of a segmented rectal probe and caudal epidural anesthesia for electroejaculation of bulls. The Canadian Veterinary Journal, 45, 235-240.

Falk, A. J., Waldner, C. L., Cotter, B. S., Gudmundson, J. \& Barth, A. D. 2001. Effects of epidural lidocaine anesthesia on bulls during electroejaculation. The Canadian Veterinary Journal, 42, 116-120.

Figueiredo, J. R. \& Molento, C. F. M. 2008. Bioética e bem-estar animal aplicados as biotécnicas reprodutivas. In: Goçalves, P. H. D., Figueiredo, J. R. \& Freitas, V. J. F. (eds.) Biotécnicas aplicadas a reprodução animal. Editora Roca, São Paulo.

Goodbourn, S., Didcock, L. \& Randall, R. E. 2000. Interferons: cell signalling, immune modulation, antiviral response and virus countermeasures. Journal of General Virology, 81, 2341-2364.

Langford, D. J., Bailey, A. L., Chanda, M. L., Clarke, S. E., Drummond, T. E., Echols, S., Glick, S., Ingrao, J., Klassen-Ross, T. \& LaCroix-Fralish, M. L. 2010. Coding of facial expressions of pain in the laboratory mouse. Nature Methods, 7, 447-449.

Marques Filho, W. C., Ferreira, J. C. P., Fugihara2, C. J., Heitmann, F. J., Ferraz, M., Monteiro, A. L., Maziero, R. R., Mártin, I. \& Oba, E. 2008. Avaliação do estresse em touros Nelore (Bos taurus indicus) submetidos à eletroejaculação. Veterinária e Zootecnia, 15, 531-541.

Mosure, W. L., Meyer, R. A., Gudmundson, J. \& Barth, A. D. 1998. Evaluation of possible methods to reduce pain associated with electroejaculation in bulls. The Canadian Veterinary Journal, 39, 504-506.

Muir, W. W. \& Gaynor, J. S. 2009. Manual de controle da dor em medicina veterinária. MedVet, São Paulo, Brasil.

Palmer, C. W. 2005. Welfare aspects of theriogenology: investigating alternatives to electroejaculation of bulls. Theriogenology, 64, 469-479.

Paranhos da Costa, M. J. R. 2004. Comportamento e bem-estar de bovinos e suas relações com a produção de qualidade. Anais dos Simpósios da 4la Reunião da Sociedade Brasileira de Zootecnia. Campo Grande, Mato Grosso do Sul.

Sandem, A.-I. \& Braastad, B. O. 2005. Effects of cow-calf separation on visible eye white and behaviour in dairy cows-A brief report. Applied Animal Behaviour Science, 95, 233239.

Sandem, A. I., Braastad, B. O. \& Bøe, K. E. 2002. Eye white may indicate emotional state on a frustration-contentedness axis in dairy cows. Applied Animal Behaviour Science, 79, 1-10.

Sandem, A. I., Janczak, A. M., Salte, R. \& Braastad, B. O. 2006. The use of diazepam as a pharmacological validation of eye white as an indicator of emotional state in dairy cows. Applied Animal Behaviour Science, 96, 177183.

Watts, J. M. \& Stookey, J. M. 2000. Vocal behaviour in cattle: the animal's commentary on its biological processes and welfare. Applied Animal Behaviour Science, 67, 15-33.

Whitlock, B. K., Coffman, E. A., Coetzee, J. F. \& Daniel, J. A. 2012. Electroejaculation increased vocalization and plasma concentrations of cortisol and progesterone, but not substance $P$, in beef bulls. Theriogenology, 78, 737-746.

\section{Article History:}

Received 3 July 2017

Accepted 9 August 2017

Available on line 4 September 2017

License information: This is an open-access article distributed under the terms of the Creative Commons Attribution License 4.0, which permits unrestricted use, distribution, and reproduction in any medium, provided the original work is properly cited. 